Original Research Article

\title{
Study of platelet parameters in bacterial infections of lower respiratory tract
}

\author{
B. Lakshmi Durga Srujana ${ }^{1}$, Yalavarthi S. ${ }^{2}$, Vasudha C.L. ${ }^{3}$ \\ ${ }^{1}$ Dr. B Lakshmi Durga Srujana, Assistant Professor, ${ }^{2}$ Dr. Sushma Yalavarthi, Professor \& Head, both authors are \\ affiliated with Department of Pathology, ${ }^{3}$ Dr. Vasudha C.L., Assistant Professor, Department of Microbiology, Mamata \\ Medical College, Khammam, Telangana, India.
}

Corresponding Author: Dr. B Lakshmi Durga Srujana, Assistant Professor, Department of Pathology, Mamata Medical College, Khammam, Telangana, India. E-mail id: srujanabld@gmail.com

\begin{abstract}
Background: Respiratory tract infections are one of the commonest bacterial infections. Platelets play an important role in inflammatory response against infectious agents. Bacterial components activate platelets to release inflammatory mediators. The changes platelets undergo during their activation are reflected in the platelet parameters. This study aims to evaluate platelet parameters in the lower respiratory tract infections. Materials \& methods: Cross-sectional study on sputum culture proven bacterial infections of the lower respiratory tract was done for three months. Platelet parameters of 94 cases were compared with 94 healthy controls. Cases were sub grouped as group A (with leucocytosis) and group B (without leukocytosis). Comparative analysis of platelet parameters between these subgroups and controls was done. $\mathrm{P}$ value was calculated and $<0.05$ was considered significant. Results: Platelet count $(\mathrm{p}<0.05)$ and plateletcrit were higher, Mean platelet volume $(\mathrm{p}<0.05)$, Platelet distribution width $(\mathrm{p}<0.001)$ and Platelet large cell ratio were lower in cases compared to controls. Group A showed significantly higher PLT $(\mathrm{p}<0.05)$ and PCT $(\mathrm{p}<0.001)$ than group B whereas the difference in other parameters was not significant. All platelet parameters were significantly altered in patients with leukocytosis on comparison with controls whereas only MPV, PDW and P-LCR showed significant change in patients without leukocytosis when compared with controls. Conclusion: Platelet parameters show variation in the lower respiratory tract infections. As they can be determined as a part of routine complete blood picture analysis, they may be considered as markers of inflammation in the diagnosis of lower respiratory tract infections.
\end{abstract}

Keywords: Leukocytosis, Mean Platelet volume, Plateletcrit, Platelet distribution width, Platelet large cell ratio, Platelet parameters.

\section{Introduction}

Role of platelets in the clotting of blood is well established. Along with the molecules necessary for aggregation, platelets also express various receptors for bacteria, complement components and many other mediators of inflammation [1]. Binding of bacterial ligands activates platelets which then show multiple responses. Antimicrobial substances are released from activated platelets, some of which opsonise bacteria whereas few others alter bacterial cell wall permeability or have direct bactericidal action [2,3].

Platelets also interact with leukocytes in bacterial clearance. Chemokines and interleukins expressed by platelets participate in recruitment and activation of leukocytes. These molecules also act on bone marrow to

Manuscript received: $10^{\text {th }}$ July 2018

Reviewed: $20^{\text {th }}$ July 2018

Author Corrected: $28^{\text {th }}$ July 2018

Accepted for Publication: $2^{\text {nd }}$ August 2018 alter the production of platelets. Activation of platelets leads to the change in their shape and size $[4,5]$. These alterations in platelet count, size and activity are reflected in platelet parameters such as platelet count (PLT), mean platelet volume (MPV), platelet distribution width (PDW), plateletcrit (PCT) and platelet large cell ratio (P-LCR).

Though all of these parameters are routinely analyzed by automated cell counters, only platelet count is generally considered in the diagnosis and management of infectious diseases.

The present study aimed to evaluate and compare platelet parameters in patients with lower respiratory tract infections and healthy controls and also to study the changes of these parameters in relation to White Blood Cell (WBC) count. 


\section{Original Research Article}

\section{Materials and Methods}

Type \& Place of study: This was a cross-sectional study conducted over a period of three months from January 2018 to March 2018 in department of Pathology, central laboratory of a tertiary care hospital with the approval of institutional ethical committee.

Study Sample: All adult patients who had come to the hospital with lower respiratory tract infections and underwent sputum culture during the study period were considered for case group and all adults who came for routine check-up during the same period were considered for control group. Data regarding age, sex, clinical diagnosis and sputum culture reports were collected from the patients. Age, sex and medical history details were collected from the individuals who came for routine health check-up.

\section{Inclusion criteria}

Cases-

- Adult patients with sputum culture proven bacterial infection of lower respiratory tract and were willing to participate in the study.

Controls-

- Healthy adults who were willing to participate in the study.

\section{Exclusion criteria}

Cases-

- Patients with lower respiratory tract infection but were bacterial culture negative.

- Sputum culture positive but not willing to participate in the study.

- Patients with diseases involving liver, renal, cardiovascular system, co-morbidities such as diabetes, hypertension, other inflammatory conditions and those on drugs which may affect platelets.

Controls-

- Adults with any co-existing medical disorder and/or on any drugs.

- Healthy adults not willing to participate in the study.

Sample collection: Total of 94 cases with positive sputum culture formed the case group of the study. 94 consecutive healthy adults who fit the study criteria were taken as control group. Informed consent was taken from both the groups and $2 \mathrm{ml}$ of venous blood was drawn into EDTA coated tubes under aseptic conditions. Complete blood count analysis was done using Fx-19 T auto hematology analyzer (Unitron Biomedicals, Bengaluru, India).

Readings were noted with emphasis on Total leukocyte count (TLC) and platelet parameters (PLT, MPV, PDW, PCT, P-LCR). Taking upper cut-off value of TLC as $11.0 \times 10^{9} / \mathrm{L}$, patients were sub grouped into group A (with leukocytosis) and group B (without leukocytosis).

Statistical methods: Statistical analyses were performed using SPSS version 20 software (SPSS Inc., Chicago, IL, USA). Qualitative variables were expressed as percentage. Quantitative variables were expressed as Mean + Standard Deviation (Mean +SD). TLC and platelet parameters were compared between cases and controls. Comparative analyses of platelet parameters between groups A and B, between group A and controls and between group B and controls were done. Independent sample $\mathrm{T}$ test was applied and $\mathrm{p}$ value of $<0.05$ was taken as statistically significant.

\section{Results}

In the period of three months, 94 patients with positive sputum culture were studied. Patients were between 21 years and 79 years of age. 50 patients $(53.19 \%)$ were males and 44 cases $(46.8 \%)$ were females. Control group included 94 healthy individuals (50 males and 44 females) between 22 and 75 years of age.

Sputum culture demonstrated gram negative organisms in 48 cases $(51.06 \%)$ while gram positive organisms were detected in 46 cases (48.93\%). Among gram negative organisms, most common isolate was Klebsiella pneumoniae (24/48 cases), followed by Pseudomonas aeruginosa (17/48 cases). 3 cases of Enterobacter spp, 2 cases of Escherichia coli and one case each of Citrobacter spp and Acinetobacter spp were also reported.

Streptococcus pneumoniae (37/46 cases) was the most common isolate among gram positive organisms. Streptococcus pyogenes was found in 7 cases and Staphylococcus aureus in 2 cases. Overall, Streptococcus pneumoniae was the most common bacteria isolated (37/94 cases, 39.36\%) followed by Klebsiella pneumoniae (24/94 cases, 25.53\%).

Mean total leukocyte count of patients was $11.18+4.99 \times 10^{9} / \mathrm{L}$ and of controls was $7.59+1.59 \times 10^{9} / \mathrm{L}$. Patients with lower respiratory tract infections showed a significant increase in total WBC count $(\mathrm{p}=0.000)$. 


\section{Original Research Article}

Mean+ SD, p value of platelet parameters of cases and controls is presented in table 1. Statistically significant difference was observed in PLT, MPV, PDW and P-LCR. Cases showed higher PLT than healthy controls where as MPV, PDW and P-LCR were lower. PCT was higher in cases, but the difference was not significant.

Table-1: Platelet parameters in cases and controls.

\begin{tabular}{|c|c|c|c|}
\hline Platelet parameters & $\begin{array}{c}\text { Cases }(\mathbf{n = 9 4 )} \\
(\text { Mean+ SD) }\end{array}$ & $\begin{array}{c}\text { Controls (n=94) } \\
\text { (Mean+ SD) }\end{array}$ & P Value \\
\hline PLT (x 10 $/ \mathrm{L})$ & $299.85+138.77$ & $257.10+60.05$ & $0.007 *$ \\
\hline MPV (fL) & $9.53+1.05$ & $9.98+1.08$ & $0.004^{*}$ \\
\hline PDW (fL) & $11.59+1.80$ & $12.90+2.01$ & 0.095 \\
\hline PCT (\%) & $0.27+0.11$ & $0.25+0.57$ & $0.002 *$ \\
\hline P-LCR (\%) & $23.58+5.90$ & $26.53+6.88$ & \\
\hline
\end{tabular}

*significant value of $\mathrm{p}(<0.05)$

Patients with leukocytosis (Group A) were 41(43.61\%) and patients without leukocytosis (Group B) were 53(56.38\%). Mean TLC in group A was $15.39+4.33 \times 10^{9} / \mathrm{L}$ and in group B was $7.92+2.32 \times 10^{9} / \mathrm{L}$, the difference being significant $(\mathrm{p}=0.000)$. Group A showed significantly higher values in PLT and PCT than group B. No significant difference was observed in MPV, PDW and P-LCR between the two groups, though higher values of PDW and lower values of MPV and P-LCR were recorded in group A. Details are shown in table 2.

Table-2: Platelet parameters of group A and group B.

\begin{tabular}{|c|c|c|c|}
\hline $\begin{array}{c}\text { Platelet } \\
\text { parameters }\end{array}$ & $\begin{array}{c}\text { Group A (n=41) } \\
\text { (Mean+ SD) }\end{array}$ & $\begin{array}{c}\text { Group B (n=53) } \\
\text { (Mean +SD) }\end{array}$ & P Value \\
\hline PLT (x 10 $/ \mathrm{L})$ & $351.60+163.81$ & $260.28+100.71$ & $0.001^{*}$ \\
\hline MPV (fL) & $9.50+1.10$ & $9.55+1.02$ & 0.836 \\
\hline PDW (fL) & $11.64+1.89$ & $11.53+1.73$ & 0.768 \\
\hline PCT (\%) & $0.31+0.13$ & $0.23+0.08$ & $0.001 *$ \\
\hline P-LCR (\%) & $23.50+6.30$ & $23.65+5.63$ & 0.902 \\
\hline
\end{tabular}

*significant value of $\mathrm{p}(<0.05)$ Group A - cases with leukocytosis, Group B - cases without leukocytosis

When groups A and B were compared with controls, cases with leukocytosis showed significant alteration in all the platelet parameters than controls while cases without leukocytosis showed significant difference only in MPV, PDW and P-LCR. TLC and PLT were higher in both the groups than controls but the difference was significant only in group A. MPV, PDW and P-LCR were significantly lower in both the groups than controls. PCT was higher in group A and lower in group B than controls (Tables 3 and 4).

Table-3: Comparison of platelet parameters between Group A (cases with Leukocytosis) and controls.

\begin{tabular}{|c|c|c|c|}
\hline $\begin{array}{c}\text { Platelet } \\
\text { parameters }\end{array}$ & $\begin{array}{c}\text { Group A (n=41) } \\
\text { (Mean+ SD) }\end{array}$ & $\begin{array}{c}\text { Controls (n=94) } \\
\text { (Mean +SD) }\end{array}$ & P Value \\
\hline PLT (x 10 $/ \mathrm{L})$ & $351.60+163.81$ & $257.10+60.05$ & $0.000^{*}$ \\
\hline MPV (fL) & $9.50+1.10$ & $9.98+1.08$ & $0.020^{*}$ \\
\hline PDW (fL) & $11.64+1.89$ & $12.90+2.01$ & $0.001^{*}$ \\
\hline PCT (\%) & $0.31+0.13$ & $0.25+0.57$ & $0.000^{*}$ \\
\hline P-LCR (\%) & $23.50+6.30$ & $26.53+6.88$ & $0.017^{*}$ \\
\hline
\end{tabular}

*significant value of p $(<0.05)$, Group A - cases with leukocytosis 


\section{Original Research Article}

Table-4: Comparison of platelet parameters between Group B (cases without leukocytosis) and controls.

\begin{tabular}{|c|c|c|c|}
\hline $\begin{array}{c}\text { Platelet } \\
\text { parameters }\end{array}$ & $\begin{array}{c}\text { Group B (n=53) } \\
\text { (Mean+ SD) }\end{array}$ & $\begin{array}{c}\text { Controls (n=94) } \\
\text { (Mean +SD) }\end{array}$ & P Value \\
\hline PLT (x 109 /L) & $260.28+100.71$ & $257.10+60.05$ & 0.811 \\
\hline MPV (fL) & $9.55+1.02$ & $9.98+1.08$ & $0.019^{*}$ \\
\hline PDW (fL) & $11.53+1.73$ & $12.90+2.01$ & $0.000^{*}$ \\
\hline PCT (\%) & $0.23+0.08$ & $0.25+0.57$ & 0.262 \\
\hline P-LCR (\%) & $23.65+5.63$ & $26.53+6.88$ & $0.010^{*}$ \\
\hline
\end{tabular}

*significant value of p $(<0.05)$, Group B - cases without leukocytosis

\section{Discussion}

Platelets have a well-established role in defensive response to infections. Changes in platelet parameters can serve as markers of platelet release and activation seen during inflammatory response. Utility of these parameters as adjunctive inflammatory markers has been studied in various acute and chronic infectious diseases.

Respiratory tract infections are one of the commonest infectious diseases affecting humans. In the present study, Streptococcus pneumoniae was the most common organism detected (39.36\%). Our findings were similar to the results of a study done on adult patients with pneumonia in north India [6]. In accordance with other studies TLC was significantly higher in our cases when compared to healthy controls $[7,8]$. Leukocytosis is one of the acute phase responses seen during infections and inflammatory disorders.

Our study revealed that platelet count was significantly higher in lower respiratory tract infections. Similar results were shown by several other studies done in infectious and inflammatory diseases $[7,9,10]$. In studies done on tuberculosis and pneumonia patients higher PLT was recorded in tuberculosis than pneumonia. Besides, patients with active tuberculosis showed higher PLT than those with inactive disease $[7,11]$.

Elevated platelet count can be explained by the induction of megakaryopoiesis. Bacterial entry into the body evokes an inflammatory reaction characterized by elevation of acute phase reactants such as interleukins (IL), of which IL-6 acts on liver to produce Thrombopoietin, a growth hormone for megakaryocytes $[5,9]$. Mean platelet volume is the most widely studied of all the platelet parameters. During infections platelets are activated and become spherical, larger which causes change in their size, shape and volume $[12,13]$. MPV in our study was significantly lower in patients compared to healthy controls. When we reviewed literature conflicting results were seen about MPV. Studies on MPV in respiratory tract infections such as pneumonia and tuberculosis revealed that MPV was lower in pneumonia compared to tuberculosis and healthy controls [8,11]. Zareifar et al [10] assessed MPV in infectious and inflammatory conditions and reported lower MPV in the active stage of disease. In a similar study done on patients with acute febrile illness majority showed low MPV and it was found to be in negative correlation with platelet count. MPV levels were relatively lower in infections caused by bacteria and mycobacteria when compared with rickettsial, parasitic and viral infections [14]. Contrarily, higher values of MPV in infections were also reported in some studies [15-17].

In severe acute infection overproduction of highly active cytokines such as tumor necrosis factor suppress megakaryopoiesis resulting in production of smaller platelets. This is complemented by inhibitory effect of bacterial cell wall lipopolysaccharides on platelet production [5,13]. Excessive consumption of larger activated platelets at the sites of inflammation can be one of the possible reasons of lower mean volume of the platelets in infectious diseases [12]. Degranulation and release of contents is followed by destruction of activated platelets, reducing the platelet count and MPV $[4,12]$. Compensatory bone marrow hyper proliferation reflected as reactive thrombocytosis is more common during chronic phases of infection [5].

Platelet distribution width gives information about variation in the volume of platelets. In our study, PDW was found to be significantly lower in cases than controls. Similarly, Nassaji et al [17] observed lower PDW in patients with pyelonephritis. Inflammatory conditions with higher PLT show lower PDW [14]. Variation of PDW with the activity of disease was also studied by some authors who demonstrated higher value in active disease and decrease in the value with 


\section{Original Research Article}

treatment [11]. Plateletcrit is a measure of volume of platelets in a unit volume of blood. It gives an idea about mass of the circulating platelets. In the present study, higher PCT was recorded in patients when compared with controls, however the difference was not statistically significant. Platelet count positively correlates with PCT [14]. Sahin et al [7] studied about PCT in tuberculosis and obtained similar results. They also concluded that plateletcrit was more significant for tuberculosis than other platelet indices. P-LCR is the proportion of platelets which are $>12 \mathrm{fL}$. P-LCR was observed to be significantly lower in cases than that of control group of our study which was in accordance to Babu et al [18] study finding of inverse relation between PLT and P-LCR. Conditions with thrombocytosis show lower P-LCR and those with thrombocytopenia show higher P-LCR $[18,19]$.

Platelet parameters were studied in relation to $\mathrm{WBC}$ count in some studies [7,20,21]. Our study revealed significantly higher values of PLT and PCT in patients who had leukocytosis than patients without leukocytosis and healthy controls. Patients without leukocytosis also had higher PLT values than healthy people but not by a significant difference. MPV, PDW and P-LCR showed no significant difference between the two sub groups of patients but on comparison of each sub group with healthy controls these parameters were found to be significantly lower. The results were similar to that of Ozturk et al [21] study on platelet parameters in leukocytosis.

\section{Conclusion}

- Platelet parameters show definite alterations in the bacterial infections of lower respiratory tract and can serve as additional markers of inflammation.

- Platelet parameters are determined by hematology cell counters as a part of complete blood picture analysis which is a routine first line investigation ordered in patients with infections. As they can be acquired without additional cost or time, studying these parameters should be considered in the diagnosis of lower respiratory tract infections.

- Though leukocytosis is a classical marker of inflammation, infections without leukocytosis also show significant change in platelet parameters making these parameters potential alternatives to determine inflammation associated with infectious diseases.

- However, considering the variation in these parameters in different types of infections, further studies are necessary to determine the exact role of these parameters in predicting the diagnosis of infectious diseases.
Contribution by authors- Dr. B Lakshmi Durga Srujana contributed to data collection and analyses, literature review, statistical analyses \& interpretation of results and preparation of manuscript. Dr. Sushma Yalavarthi contributed to the conception \& design of the study, analyses \& interpretation of results and manuscript editing. Dr. Vasudha CL contributed to data acquisition, statistical analyses and manuscript editing.

Funding: Nil, Conflict of interest: None initiated Permission from IRB: Yes

\section{What this study adds}

- Along with leukocytosis and thrombocytosis plateletcrit also increases in respiratory tract infections.

- Mean platelet volume, platelet distribution width decrease in infections even when there is no leukocytosis.

\section{References}

1. Hamzeh-Cognasse H, Damien P, Chabert A, et al. Platelets and infections - complex interactions with bacteria. DOI:10.3389/fimmu.2015.00082

2. Krauel K, Weber C, Brandt S. et al. Platelet factor 4 binding to lipid A of Gram-negative bacteria exposes PF4/heparin-like epitopes. doi: 10.1182/blood-2012-06434985. Epub 2012 Aug 31.

3. Yang D, Chen Q, Hoover DM, et al. Many chemokines including CCL20/MIP- 3alpha display antimicrobial activity. J Leukoc Biol. 2003 Sep; 74 (3): 448-55.

4. Yeaman MR. Platelets in defense against bacterial pathogens. doi: 10.1007/s00018-009-0210-4. Epub 2009 Dec 15.

5. Klinger MH, Jelkmann W. Role of blood platelets in infection and inflammation. DOI:10.1089/10799 900 260286623

6. Para RA, Fomda BA, Jan RA, et al. Microbial etiology in hospitalized North Indian adults with community-acquired pneumonia. DOI: 10.4103/ lungindia. lungindia_288_17

7. Sahin F, Yazar E, Yildiz P. Prominent features of platelet count, plateletcrit, mean platelet volume and platelet distribution width in pulmonary tuberculosis. Multidiscip Respir Med. 2012;7(1):38. doi: 10.1186/ 2049-6958-7-38. Google/Pubmed 


\section{Original Research Article}

8. Karadag-Oncel E, Ozsurekci Y, Kara A, et al. The value of mean platelet volume in the determination of community acquired pneumonia in children. DOI:10.1186/1824-7288-39-16

9.Unsal E, Aksaray S, Köksal D, Sipit T. Potential role of interleukin 6 in reactive thrombo-cytosis and acute phase response in pulmonary tuber-culosis. DOI: 10. 1136/pgmj.2004.030544

10. Zareifar S, Farahmand Far MR, Golfeshan F, Cohan $\mathrm{N}$. Changes in platelet count and mean platelet volume during infectious and inflammatory disease and their correlation with ESR and CRP. DOI:10.1002/jcla.21673

11. Tozkoparan E, Deniz O, Ucar E, et al. Changes in platelet count and indices in pulmonary tuberculosis. DOI:10.1515/CCLM.2007.194

12. Afyon M, Artuk C. Could Mean Platelet Volume be a useful marker for infectious diseases? A review of literature. Med SCi.2016;5(4):1059-62. doi: 10.5455/ medscience. 2016.05.8460. Google

13. Bath PM, Butterworth RJ. Platelet size: measurement, physiology and vascular disease. Blood Coagul Fibrinolysis 1996;7(2):157-61. Google/Pubmed

14. Viswanathan S, Saravanakumari V. Are platelet indices useful in diagnosis if tropical acute febrile illnesses? Journal of Local and Global Health Science. 2016:3. doi: 10.5339/jlghs.2016.3. Google

15. Ladhani S, Lowe B, Cole AO, Kowuondo K, Newton CR. Changes in white blood cells and platelets in children with falciparum malaria: relationship to disease outcome. Br J Haematol 2002;119(3):839-47. doi:10.1046/j.1365-2141.2002.03904.x.Google/Pubmed

16. Cho SY, Jeon YL, Kim W, et al. Mean platelet volume and mean platelet volume/platelet count ratio in infective endocarditis. DOI: 10.3109/09537104. 2013. 857394.

17. Tekin $\mathrm{M}^{1}$, Konca $\mathrm{C}$, Gulyuz $\mathrm{A}$, et al. Is the mean platelet volume a predictive marker for the diagnosis of acute pyelonephritis in children? DOI:10.1007/ s101 57-014-1049-z

18. Babu E, Basu D. Platelet large cell ratio in the differential diagnosis of abnormal platelet counts. Indian J Pathol Microbiol.2005;47(2):202-5.

19. Yalavarti S, Halder NR, Pagidikalava A. Utility of Platelet Indices in diagnosing the underlying cause of thrombocytopenia due to accelerated platelet destruction. Annals of pathology and laboratory medicine. 2018; 5 (4):296-300. doi: 10.21276/APALM. 1717. Google

20. Santimone I, Di Castelnuovo A, De Curtis A, et al. White blood cell count, sex and age are major determinants of heterogeneity of platelet indices in an adult general population: results from the MOLI-SANI project. DOI: 10.3324/haematol.2011.043042

21. Ozturk N, Baygutalp NK, Bakan E, Altas GF, Polat $\mathrm{H}$, Dorman E. Changes in platelet parameters in leukocytosis. Pan African Medical Journal. 2016; 24: 185. doi: 10.11604/ pamj. 2016.24.185.7510. Google/ Pubmed.

\section{How to cite this article?}

B.Lakshmi Durga Srujana, Yalavarthi S, Vasudha C.L. Study of platelet parameters in bacterial infections of lower respiratory tract. Trop J Path Micro 2018;4(4):308-313.doi:10. 17511/ jopm. 2018.i4.02 